Review

\title{
Strongyloidiasis in Solid Organ Transplantation
}

Ashrit Multani ${ }^{*}$, Stanley Deresinski

Division of Infectious Diseases \& Geographic Medicine, Department of Medicine, Stanford University School of Medicine, Stanford, California, USA; E-Mails: amultani@stanford.edu; polishmd@stanford.edu

* Correspondence: Ashrit Multani; E-Mail: amultani@stanford.edu

Academic Editor: Maricar Malinis

Special Issue: Diagnosis and Management of Infections in Solid Organ Transplant Recipients

OBM Transplantation

2018, volume 2, issue 4

doi:10.21926/obm.transplant.1804035
Received: October 31, 2018

Accepted: December 24, 2018

Published: December 27, 2018

\begin{abstract}
Strongyloides stercoralis, an intestinal parasitic nematode (roundworm), infects more than 100 million people worldwide. While most infected immunocompetent persons are either asymptomatic or experience only mild, intermittent symptoms, immunosuppressed individuals, including those undergoing solid organ transplantation, are at increased risk for developing the frequently fatal hyperinfection syndrome. Donor-derived strongyloidiasis has recently become increasingly recognized and reported but current donor screening practices leave substantial room for improvement. Knowledge that available standard diagnostic tests have suboptimal performance characteristics has led to the development of newer diagnostic assays including use of polymerase chain reactions. Ivermectin has been considered the recommended therapeutic agent but current evidence suggests that it may be less effective than previously believed. Because of the increasing global performance of solid organ transplantation, together with the global presence of the parasite, this infection is of universal interest. While some management issues are yet to be resolved, fatal outcomes from Strongyloides hyperinfection syndrome can be prevented by appropriate pre-transplantation screening and treatment of the solid organ donor and/or the recipient.
\end{abstract}

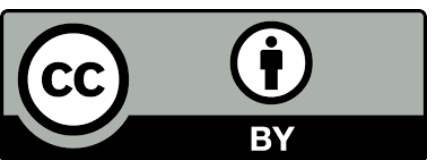

(C) 2018 by the author. This is an open access article distributed under the conditions of the Creative Commons by Attribution License, which permits unrestricted use, distribution, and reproduction in any medium or format, provided the original work is correctly cited. 


\section{Keywords}

Strongyloidiasis; Strongyloides stercoralis; hyperinfection syndrome; solid organ transplantation; donor-derived infection; donor-derived strongyloidiasis

\section{Introduction}

Strongyloidiasis is caused by infection with Strongyloides stercoralis, an intestinal parasitic nematode (roundworm) that infects more than 100 million people worldwide [1, 2]. While most infected immunocompetent persons are either asymptomatic or experience only mild, intermittent symptoms, immunosuppressed individuals, including those undergoing solid organ transplantation (SOT), are at increased risk for developing the frequently fatal hyperinfection syndrome.

The occurrence of donor-derived strongyloidiasis has recently become increasingly recognized and reported [3-11]. Standard diagnostic tests have long been acknowledged to have suboptimal performance characteristics and this has led to the development of newer diagnostic assays including use of the polymerase chain reaction (PCR) [12-16]. Ivermectin has been considered the recommended therapeutic agent but recommended dosing regimens have not been standardized and its efficacy may be less than previously believed [17-20]. Because of the global presence of both S. stercoralis and SOT, this infection is of universal interest. While some management issues are yet to be resolved, fatal outcomes from Strongyloides hyperinfection syndrome can be prevented by appropriate pre-transplantation screening and treatment.

\section{Epidemiology}

S. stercoralis is endemic in rural parts of tropical and subtropical areas such as Southeast Asia, Latin America, the Caribbean islands, sub-Saharan Africa, and southern and eastern Europe with an overall prevalence greater than $80 \%$ in some regions $[1,2,21,22]$. In the US, S. stercoralis is endemic in rural areas of the southeastern region with an estimated prevalence of up to $6.1 \%$ and has also been sporadically found in the Appalachian region (eastern Tennessee, Kentucky, and West Virginia) $[2,23,24]$. The highest infection rates in the US are seen in immigrants and refugees from endemic areas in addition to military personnel (especially World War II and Vietnam War veterans) who served in endemic areas [2].

S. fuelleborni, another species of the same genus previously thought to infect only non-human primates, has been discovered to infect humans sporadically in Africa, Papua New Guinea, and Thailand $[2,25,26]$. Whether or not this species has any medical importance in adults or in SOT recipients is unknown. Also unknown is whether there are any noteworthy pathophysiologic differences between S. stercoralis and S. fuelleborni but the former is believed to be far more dangerous because of its potential for autoinfection, a phenomenon not known to occur with the latter [27]. Multiple other species such as S. procyonis and S. ransomi have also been identified but have not to date been recognized to naturally infect humans, although unreproducible experimental infections have been reported [2, 28, 29]. 


\section{Biology, Life Cycle, \& Pathophysiology}

The Strongyloides life cycle is more complex than most other nematodes because it can alternate between free-living and parasitic cycles and because of its potential for autoinfection (Figure 1, image and information courtesy of Centers for Disease Control and Prevention and DPDx). Infective filariform larvae enter the host by penetrating through the skin. The filariform larvae migrate into the small intestine by various random routes. They can reach the lungs by hematogenous and lymphatic migration or they can reach the small intestine directly via connective tissues. If migrating through the lungs, they penetrate into the alveoli, ascend the tracheobronchial tree, and are swallowed. The filariform larvae molt twice in the small intestine and mature into adult female worms that can live up to 5 years. The adult female worms burrow into the duodenal and jejunal mucosal epithelium where they produce eggs by parthenogenesis and deposit them. Eggs are produced asexually since parasitic adult males do not exist. After the eggs hatch in the mucosa, the noninfective rhabditiform larvae migrate to and develop within the bowel lumen. The rhabditiform larvae are generally passed in the feces but can also cause autoinfection. This cycle from skin penetration to fecal passage usually takes 18 to 28 days to occur. If the rhabditiform larvae are excreted with feces onto soil in a warm, humid climate, they molt and develop into either filariform larvae directly or free-living adult males and females. If the latter occurs, the free-living adults mate and produce eggs from which rhabditiform larvae hatch. These rhabditiform larvae eventually become filariform larvae.

In autoinfection, S. stercoralis can complete its life cycle entirely within the human host. The rhabditiform larvae mature into filariform larvae in the large intestine and penetrate either through the intestinal mucosa (internal autoinfection) or the perianal skin (external autoinfection). In either situation, the filariform larvae can disseminate randomly to other organs throughout the body. The burden of adult worms can increase significantly during a single autoinfection cycle. Larval transformation within the gastrointestinal tract can be accelerated by bowel pathology (e.g., constipation, diverticulae, hypomotility) or corticosteroids. Autoinfection is the most likely explanation for chronic infection in the absence of exogenous reinfection (including those persons who have not been in an endemic area for multiple decades), hyperinfection syndrome, and person-to-person transmission [28, 30,31]. Autoinfection is limited by an intact host immune response but a low level of autoinfection may allow for chronic infections [32]. The host immune response to strongyloidiasis, as with other metazoan parasites, is mainly Th2 with associated elevated levels of IL-4, IL-5, IL-10, IL-13, IgE, and eosinophils in the peripheral blood [33, 34]. In hosts with depressed cell-mediated immunity, particularly those receiving corticosteroids, autoinfection may lead to potentially fatal hyperinfection syndrome [35]. Autoinfection has only been recognized to occur in one other helminth infection, that caused by Capillaria philippinensis. 


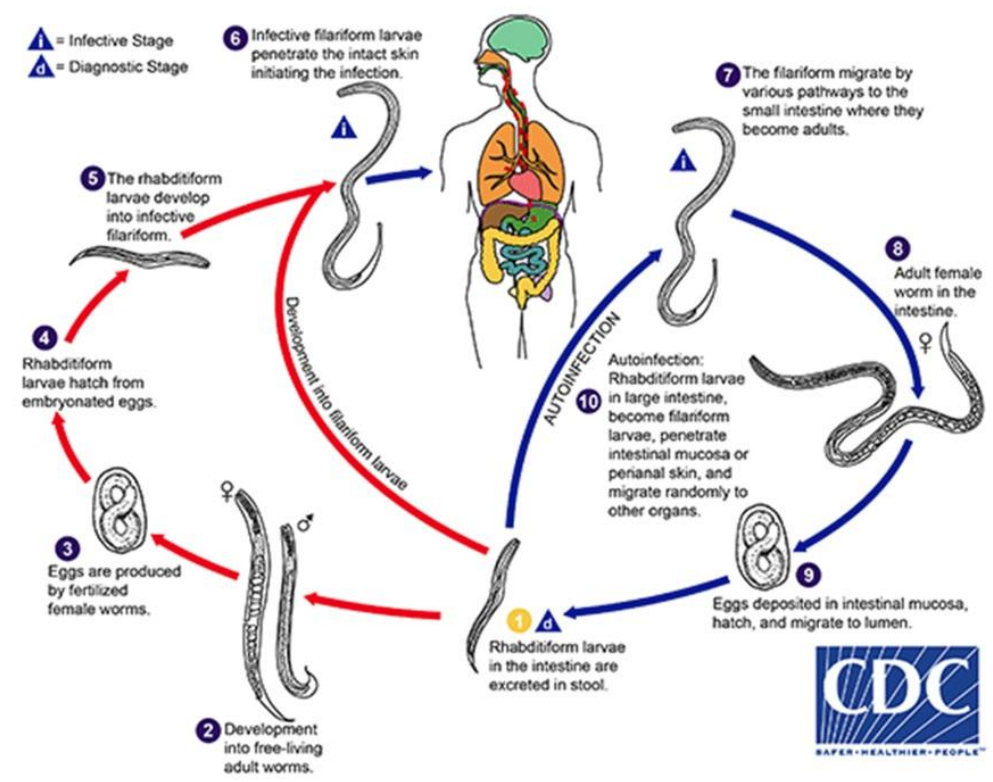

The Strongyloides life cycle is more complex than that of most nematodes with its alternation between free-living and parasitic cycles, and its potential for autoinfection and multiplication within the host. Two types of cycles exist: Free-living cycle: The rhabditiform larvae passed in the stool (1) (see "Parasitic cycle" below) can either become infective filariform larvae (direct development) 6 or free living adult males and females (2) that mate and produce eggs 3 from which rhabditiform larvae hatch $\mathbf{4}$. and eventually become infective filariform larvae $\boldsymbol{\Theta}$. The filariform larvae penetrate the human host skin to initiate the parasitic cycle (see below) $\boldsymbol{\Theta}$. Parasitic cycle: Filariform larvae in contaminated soil penetrate the human skin $\boldsymbol{\Theta}$, and by various, often random routes, migrate into the small intestine 7 . Historically it was believed that the L 3 larvae migrate via the bloodstream to the lungs, where they are eventually coughed up and swallowed. However, there is also evidence that L 3 larvae can migrate directly to the intestine via connective tissues. In the small intestine they molt twice and become adult female worms $\mathbf{8}$. The females live threaded in the epithelium of the small intestine and by parthenogenesis produce eggs $\boldsymbol{\Theta}$, which yield rhabditiform larvae. The rhabditiform larvae can either be passed in the stool (9) (see "Free-living cycle" above), or can cause autoinfection (1). In autoinfection, the rhabditiform larvae become infective filariform larvae, which can penetrate either the intestinal mucosa (internal autoinfection) or the skin of the perianal area (external autoinfection); in either case, the filariform larvae may disseminate throughout the body. To date, occurrence of autoinfection in humans with helminthic infections is recognized only in Strongyloides stercoralis and Capillaria philippinensis infections. In the case of Strongyloides, autoinfection may explain the possibility of persistent infections for many years in persons who have not been in an endemic area and of hyperinfections in immunosuppressed individuals.

Figure 1 Life cycle of Strongyloides stercoralis. Image and information courtesy of Centers for Disease Control and Prevention and DPDx.

\section{Risk Factors}

Strongyloidiasis is acquired from skin contact with contaminated materials, most commonly by walking barefoot on soil that has been contaminated with human feces harboring infective filariform larvae. SOT recipients may have chronic infection for decades that only becomes clinically apparent in the setting of post-transplantation immunosuppression but they may also acquire de novo primary infection if they are exposed to the parasite post-transplantation. Chronic infection can persist for more than 75 years, highlighting the importance of obtaining a thorough lifetime travel history of both the donor and recipient [31]. Person-to-person transmission has been reported to be a cause of institutional outbreaks in long-term care facilities [36, 37]. Close contact with the respiratory secretions of a patient with hyperinfection syndrome including pulmonary strongyloidiasis may transmit filariform larvae [38]. Sexual transmission of S. stercoralis has been seen in men who have sex with men [39]. Donor-derived infections in SOT recipients have also been described [3-11]. Infant infection with $S$. fuelleborni has been suspected to be acquired through the breastmilk of nursing mothers [27, 40]. 
Hyperinfection syndrome develops in patients with cell-mediated immune dysfunction [35]. SOT recipients are at lifelong risk because of their requisite immunosuppression. Corticosteroid use, even with courses as short as 6 days, is the most common and most specific risk factor for transforming chronic infection to hyperinfection but the underlying mechanisms for this development are not well understood [41-43]. One hypothesis suggests that corticosteroids increase risk for hyperinfection by acting on the host immune system particularly through suppression of eosinophil and lymphocyte activation [35]. Other theories propose that corticosteroids act directly on the parasites either by spurring larval transformation of rhabditiform to filariform larvae or by stimulating barren adult female worms to produce eggs again [44-46]. Lending supportive evidence to the parasite activation premise is that $S$. stercoralis larvae were discovered to have steroid/thyroid hormone receptors through which life cycle acceleration may be mediated [47]. Besides corticosteroids, immunosuppressives commonly used in SOT that increase risk for Strongyloides hyperinfection include antithymocyte globulin, azathioprine, and mycophenolate mofetil $[35,48]$. Other identified risk factors for hyperinfection include hematopoietic cell transplantation, cytotoxic therapies, anti-tumor necrosis factor receptor agents, congenital immunodeficiency, malnutrition, alcoholism, solid tumor and hematologic malignancy, hypogammaglobulinemia, gut hypomotility, hypochlorhydria, diabetes mellitus, human T-lymphotropic virus type I (HTLV-I), and human immunodeficiency virus and acquired immunodeficiency syndrome $[35,43,48]$.

\section{Donor-Derived Infection}

Donor-derived strongyloidiasis in SOT is rare but has been reported to occur [3-11]. The first possible transmission was reported in 1981 when two kidney transplant recipients developed hyperinfection syndrome after receiving allografts from the same deceased donor [3]. Thus far, the vast majority of donor-derived infections have been reported in kidney transplantation followed by liver, kidney-pancreas, heart, intestine, and pancreas transplantation [10]. Notably, most of these infections have been described within the last 10 years. Donor-derived infections are also being reported in non-endemic areas. One possible explanation for these emerging trends is increased globalization and expanding availability of SOT including in Strongyloides endemic areas [10]. An illustrative example of this recently occurred in Norway (where infection with $S$. stercoralis is very rare) after two kidney transplant recipients developed donor-derived strongyloidiasis after receiving their allografts from the same deceased donor who was originally born in an endemic area [11]. Another interesting hypothesis is related to the trend over the past 2 decades to switch maintenance calcineurin inhibitor from cyclosporine to tacrolimus. It has been suggested that this switch away from cyclosporine may have led to an increased incidence of donor-derived infection as cyclosporine has been shown to have antihelminthic properties against S. stercoralis and may have incidentally provided protection previously [49]. The majority of reported cases developed clinical manifestations several weeks to months after undergoing SOT, with a median time to symptom onset of 72 days (range, 30 to 270 days) [8-10,50]. The presentations of donor-derived Strongyloides infection are similar to those in other clinical settings and will be discussed in detail later.

Universal living or deceased donor screening for Strongyloides infection is not recommended by the American Society of Transplantation (AST), The Organ Procurement and Transplantation 
Network (OPTN), or United Network for for Organ Sharing (UNOS). In February 2013, OPTN mandated that transplant centers develop a written protocol for identifying and testing potential living donors at risk for transmissible seasonal or geographically defined endemic disease as part of the medical evaluation process [22]. The AST Infectious Diseases Community of Practice recommended against screening all potential living donors and instead advocated for targeted risk-based screening of the following individuals: persons who were born in or lived in tropical or subtropical countries where sanitation conditions are substandard, persons with unexplained eosinophilia and travel to an endemic area, persons born in the US who have significant exposure to soil in Appalachia or the southeastern US, and persons reporting a prior history of Strongyloides infection [22, 51]. A 2016 survey of all 58 US organ procurement organizations (OPOs) revealed that only $6(10 \%)$ currently screen donors for strongyloidiasis [52]. Among the 52 OPOs who are not currently screening, 31 (60\%) have not considered screening and 6 (11\%) considered screening and decided not to screen [52]. Clearly, there is a consequential need for improvement in this area. A suggested approach for the prevention of donor-derived strongyloidiasis in SOT is summarily presented in Figure 2.

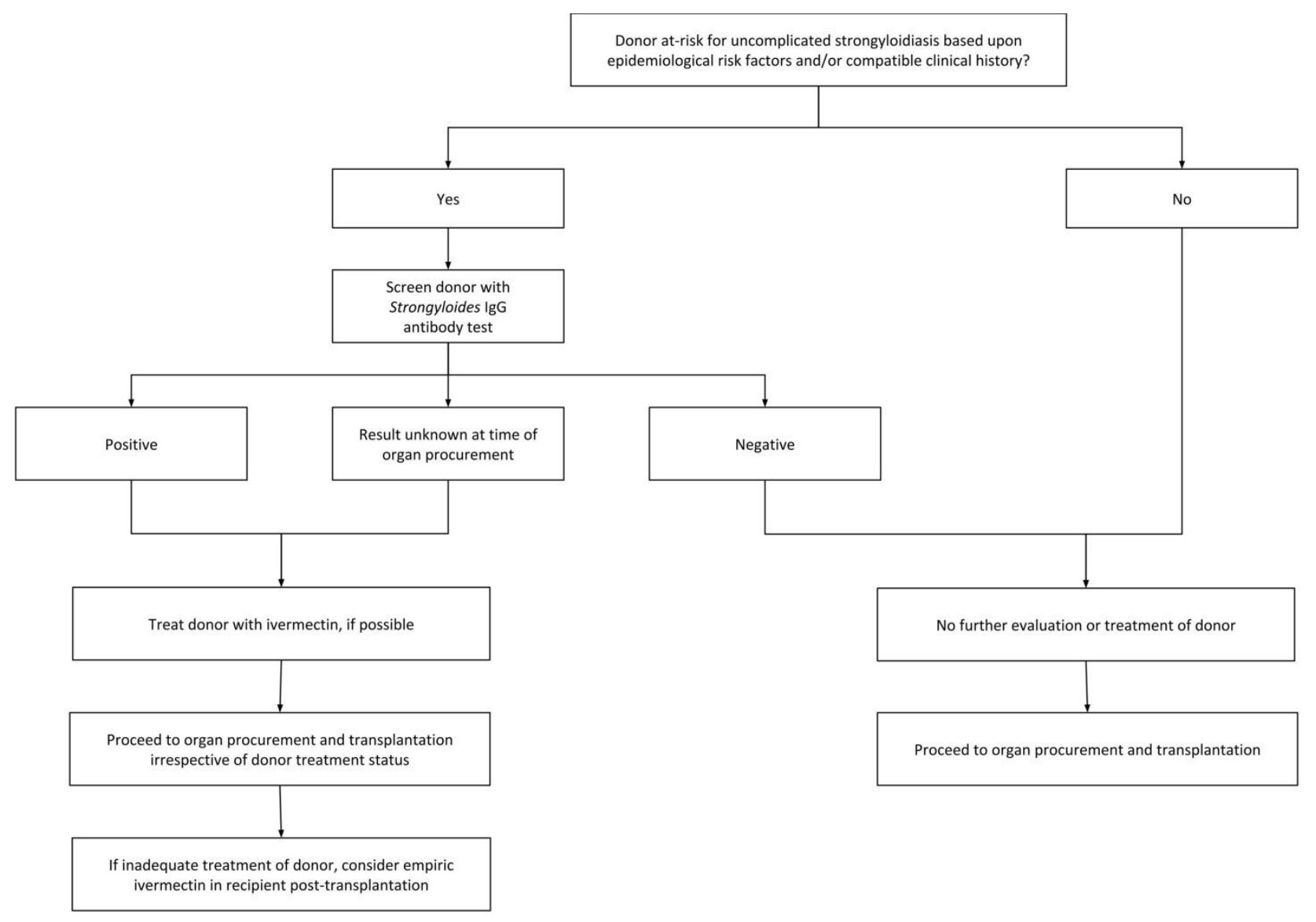

Figure 2 Suggested approach for prevention of donor-derived infection in solid organ transplantation.

Serology is the preferred screening test for Strongyloides infection. Stool microscopy can be considered but its poor sensitivity limits its utility [12-15]. The role of donor stool PCR testing for $S$. stercoralis has yet to be established $[14,20]$. The optimal timing of living donor screening has not been identified but it seems reasonable to test at the time of donor identification and initial 
testing assuming that the donor is not going to be at risk for acquiring Strongyloides infection after screening [22]. This would allow sufficient time for treatment if indicated. Following treatment, there is no need for follow-up donor testing as a test-of-cure prior to organ procurement unless the donor is re-exposed [22]. Neither treated nor untreated donor intestinal Strongyloides infection should be considered a contraindication to organ procurement since the average time from transplant to donor-derived infection onset is delayed and because safe and effective antihelminthic regimens are available for the SOT recipient [8-10, 50]. Organs should not be procured from donors suspected of having disseminated strongyloidiasis or hyperinfection syndrome.

If the infected donor is appropriately treated prior to organ procurement (despite the unlikelihood that complete parasitological cure may be achievable), no specific treatment or monitoring of the SOT recipient is required as the probability of transmission is expected to be low in untreated donors and negligible in treated donors [20,22]. If the donor cannot be treated or is inadequately treated prior to procurement, the SOT recipient should receive ivermectin under expert guidance as soon as possible post-transplantation to avert adverse outcomes, a strategy which has been proven to be effective in preventing strongyloidiasis-related morbidity and mortality $[8,9,22]$. All SOT recipients of organs from Strongyloides-infected donors should have close clinical monitoring in the post-transplantation period for any evidence of donor-derived infection [22]. In the absence of clinical evidence of strongyloidiasis, no additional serological or microbiological diagnostics are indicated [22]. If the SOT recipient develops clinical evidence of donor-derived strongyloidiasis, appropriate diagnostic evaluation and/or empiric treatment should be considered under the guidance of expert consultation [22]. It is crucial to note that absence of eosinophilia and/or negative serological testing can be falsely reassuring and do not rule out the possibility of strongyloidiasis. Eosinophilia may not be present (particularly if the patient is receiving corticosteroids) and serologies may not be reliable in the SOT recipient due to their net state of immunosuppression. Treatment regimens will have to be individualized based on the specific clinical syndrome. Detailed discussion regarding diagnostic modalities and antihelminthic regimens will be discussed later.

If there is a concern for donor-derived Strongyloides infection, centers that have transplanted SOT recipients with organs procured from the same donor should communicate effectively and efficient with each other [8]. OPTN encourages OPOs and transplant centers to communicate through its Patient Safety System, ideally within 24 hours of suspicion or confirmation of transmission [8].

\section{Clinical Manifestations}

Symptomatic strongyloidiasis can present as various clinical syndromes: acute, chronic, disseminated, and hyperinfection. The majority of patients infected with S. stercoralis either do not experience noteworthy symptoms or are asymptomatic. When symptomatic, patients may have mild gastrointestinal, pulmonary, or cutaneous manifestations that may occur intermittently for years. Because larvae can disseminate to virtually any organ, a wide variety of other manifestations have been reported to be due to strongyloidiasis [35].

In acute strongyloidiasis, local reactions (sometimes termed ground itch) can occur at the site of entry of the infective filariform larvae almost immediately and persist for weeks [53, 54]. These 
manifestations can include edema, erythema, petechiae, serpiginous or urticarial tracts, and severe pruritus [54]. As larvae migrate through the lungs a few days later, a bronchitis-like clinical picture with cough and tracheal irritation may ensue [53]. As these larvae are swallowed and migrate to the gastrointestinal tract about 2 weeks after infection onset, diarrhea, constipation, abdominal pain, and anorexia may follow [53]. These symptoms and their timeline are based on experimental human infections with hundreds of larvae and likely overestimate the severity and chronology of natural infections [35].

Chronic Strongyloides infection is most often asymptomatic [28]. Chronic intermittent vomiting, diarrhea, constipation, epigastric pain resembling peptic ulcer pain, and borborygmus are the most common gastrointestinal symptoms [35]. Ileus, intestinal obstruction, and gastrointestinal bleeding have occurred after a marked increase in larval burden and could be considered a manifestation of hyperinfection if large numbers of filariform larvae are detected in the stool contemporaneously [35]. Distinctive cutaneous manifestations including pruritis ani, urticaria, and larva currens are associated with chronic infection and primarily involve the buttocks, perineum, and thighs [30, 35]. Larva currens (Latin for "racing larva"), due to intradermal parasitic migration and pathognomonic for strongyloidiasis, is so named because of its relative speed (advancing as much as $1 \mathrm{~cm}$ in 5 minutes and 5 to $15 \mathrm{~cm}$ per hour) and is also notable for its perianal involvement, wide zone of urticaria, and evanescence, factors that help distinguish it from cutaneous larva migrans [55]. Pulmonary symptoms are uncommon [28]. Recurrent asthma, especially if induced or exacerbated by corticosteroids, has also been attributed to, and should heighten suspicion for, chronic S. stercoralis infection $[35,56]$. It has been proposed that chronic strongyloidiasis can increase the risk of adult T-cell leukemia/lymphoma in patients with HTLV-I [57].

Disseminated strongyloidiasis refers to larval migration to organs outside of the traditional autoinfection cycle [35]. Disseminated infection does not necessarily confer greater infection severity and the term should not be used interchangeably with hyperinfection syndrome [35]. Disseminated infection can occur without manifestations of hyperinfection syndrome and hyperinfection syndrome can occur without larvae being detected outside the pulmonary autoinfection cycle route [35]. Nephrotic syndrome has been attributed to disseminated strongyloidiasis in a single case report [58]. S. stercoralis has also been reported to cause eosinophil-predominant hemorrhagic pericardial effusion [59]. Larvae have also been reported to disseminate to the mesenteric lymph nodes, gallbladder, liver, diaphragm, pancreas, ovaries, skeletal muscle, and central nervous system [35].

Hyperinfection syndrome is an often overwhelming, life-threatening infection resulting from accelerated autoinfection that is usually but not always due to cell-mediated immune dysfunction $[35,43]$. SOT recipients are at increased risk for hyperinfection syndrome because of their requisite immunosuppressives. The diagnosis of hyperinfection syndrome is predicated on the idea that clinical manifestations result from increased larval migration [35]. The development or exacerbation of gastrointestinal and pulmonary symptoms in conjunction with the simultaneous detection of increased numbers of larvae in stool and/or respiratory tract specimens is characteristic of hyperinfection syndrome [35]. Hyperinfection syndrome can occur with both disseminated and non-disseminated strongyloidiasis [35].

Clinical manifestations of hyperinfection syndrome are widely variable [35]. There is no "typical" time to symptom onset and it may be acute or insidious [35]. Constitutional symptoms 
such as fever and chills may not be present. Fatigue, weakness, or total body pain may be subtle, nonspecific findings [35]. Some case reports do not describe any gastrointestinal symptoms whereas others have described patients with abdominal pain (sometimes epigastric in location, often crampy or bloating in nature), watery diarrhea, gastrointestinal bleeding, constipation, anorexia, weight loss, nausea, vomiting, dysphagia, and/or odynophagia either in isolation or any combination [35]. The clinical picture may be mistaken for ulcerative colitis [60]. Third-spacing in the form of peripheral edema, ascites, and anasarca may result from protein-losing enteropathy [35]. Pulmonary symptoms are similarly varied. Some patients do not experience any pulmonary symptoms. Others may have cough, wheezing, choking sensation, hoarseness, chest pain (that is sometimes pleuritic), hemoptysis (which may be massive especially in the setting of diffuse alveolar hemorrhage [DAH]), dyspnea, tachypnea, acute respiratory distress syndrome (ARDS), and respiratory failure $[35,61]$. ARDS, DAH, and/or radiographic appearance of diffuse bilateral ground glass opacities (GGO) on chest computed tomography (CT), especially in a patient with epidemiologic risk who is receiving corticosteroids, should heighten suspicion for hyperinfection syndrome [32, 35, 41]. Larva currens is the most frequently seen dermatologic manifestation of hyperinfection syndrome, primarily occurring on the skin of the lower trunk, thighs, and buttocks $[35,55]$. Petechial and purpuric lesions can also occur in these same regions [35]. Periumbilical thumbprint purpura is characteristic [7]. Cutaneous vasculitis may be seen [35]. Enteric organisms may enter the bloodstream through gastrointestinal ulcers or presumably by transport on the surface and in the gut of migrating larvae although the latter mechanism has never been proven [41]. Gram-negative sepsis may lead to disseminated intravascular coagulation and its associated skin findings [35]. Meningeal symptoms and signs are the most common manifestation of central nervous system strongyloidiasis with or without bacterial coinfection; brain abscesses may also occur [35]. S. fuelleborni can produce life-threatening generalized edema and respiratory distress, termed "swollen belly syndrome," in infants [25].

\section{Diagnosis}

There are multiple diagnostic tools available for the detection of Strongyloides infection, with the utility of each dependent upon the host and the clinical syndrome. A combination of currently available diagnostic tests may be required in order to achieve maximum sensitivity and specificity. In general, chronic strongyloidiasis, in particular, is difficult to diagnose by clinical specimen examination because of low parasite loads and irregular larval output in the absence of hyperinfection syndrome [62]. Furthermore, it is difficult to assess the relative performance characteristics of the various diagnostic modalities because of the lack of a high-quality gold standard for comparison [34]. Some laboratory parameters may yield clues to strongyloidiasis but are unfortunately nonspecific. Leukocytosis is variable and should not be expected in the setting of immunosuppression. Eosinophilia is not universally seen but it may be mild and the only hint of a parasitic infection, portending a more favorable prognosis when present $[35,62]$. Eosinophilia may be absent or suppressed in the setting of a concomitant bacterial infection, corticosteroid administration, or hyperinfection [35]. Protein-losing enteropathy may lead to new-onset or worsening hypoalbuminemia [35]. Hypokalemia and other electrolyte disturbances may result from gastrointestinal pathology [35]. Hyponatremia may be seen in patients with meningitis [35]. Patients with HTLV-I and strongyloidiasis coinfection may or may not have elevated IgE levels [63, 
64]. Fecal occult blood testing can be positive in the setting of gastrointestinal infection leading to bleeding $[35,61,62]$.

In uncomplicated cases of strongyloidiasis (i.e., those cases without disseminated infection or hyperinfection syndrome), the sensitivity of wet mount examination of stool specimens for larvae is notoriously poor due to the low intestinal burden and intermittent excretion of larvae [62]. In more than two-thirds of cases, there are 25 or fewer larvae per gram of stool [62]. A single stool examination has a sensitivity as low as 30\% [62]. The sensitivity improves to $50 \%$ with 3 stool examinations and can approach $100 \%$ if 7 serial stool samples are examined $[30,62,65]$. Because of this method's low sensitivity, a negative test does not exclude the diagnosis and cannot be solely relied upon [35]. Other techniques including direct smear of feces in saline-Lugol iodine stain, Baermann concentration, formalin-ethyl acetate concentration, and Harada-Mori filter paper culture are much more sensitive than single stool-smears but are rarely standard procedures in clinical parasitology laboratories [62]. In hyperinfection syndrome, organism detection becomes relatively easier, directly correlating with the increased larval burden as the autoinfection cycle accelerates [62]. In disseminated strongyloidiasis, the organism can be found in a wide variety of clinical specimens depending on the organ involved. Larvae can be identified in sputum, bronchoalveolar lavage fluid, bronchial washings and brushings, lung biopsies, pleural fluid, pericardial fluid, urine, ascitic fluid, surgical drainage fluid, cerebrospinal fluid, blood smears, and skin biopsy specimens $[35,59,62]$.

Multiple other diagnostic techniques have been employed, including the agar culture method which is the most sensitive of the available concentration methods $[28,48]$. In the agar culture method, the stool specimen is placed on a nutrient agar plate and incubated for at least 48 hours [62]. As the larvae crawl over the agar, enteric bacteria are carried with them and create visible serpiginous tracks of bacterial growth on the culture media [62]. This technique is labor-intensive and time-consuming but it has been reported to have $96 \%$ sensitivity and perform $>4$ times more efficiently than the direct smear method [62].

Several serum antibody detection tests using a variety of antigens have been developed over many years [34]. Serologic diagnosis of strongyloidiasis by enzyme-linked immunosorbent assay (ELISA) is often more sensitive than microscopic examination of clinical specimens but cannot distinguish between active or prior infection and may not be available worldwide [51]. There are multiple ELISA serologies offered by commercial laboratories with variable reliability. ELISA has 80 to $95 \%$ sensitivity and $90 \%$ specificity in immunocompetent individuals [51]. In immunocompromised patients, particularly those with hematologic malignancy, sensitivity decreases to $68 \%$ but specificity remains $89 \%[51,66]$. These performance characteristics likely do not reflect the true utility of the test as they are are often calculated in comparison to the notoriously suboptimal stool examination [34]. False-positive serologies have been reported due to cross-reactivity with other helminth infections and should be taken into consideration depending on the epidemiologic risk of the individual being tested [34]. False-negatives can occur in the setting of post-transplant immunosuppression. Overall, it is reasonable to surmise that serology overestimates the burden of disease whereas conventional microbiological techniques underestimate it [34].

Luciferase immunoprecipitation systems (LIPS) are newer immunologic assays that have been shown to be superior to other types of immunoassays with sensitivity and specificity approaching $100 \%$ [48]. These assays rely on rapid immunoprecipitation systems to detect IgG antibodies to a 
recombinant Strongyloides antigen (NIE) and S. stercoralis immunoreactive antigen (SsIR) in a high-throughput format [48]. Another potential advantage is that this test reverts to seronegativity after curative treatment [48].

Multiple molecular methods using PCR are under development but are not yet widely available for clinical use $[14,15,67,68]$. Strongyloides-specific PCR-based methods can have sensitivity and specificity reaching $100 \%$ and can achieve a diagnosis up to one month earlier when compared to conventional microbiological methods [15]. S. stercoralis has also been reported to be incidentally diagnosed by broad-range $28 \mathrm{~S}$ rDNA gene sequencing [69]. While molecular methods usually entail higher initial and overhead costs, they prove to be cost-effective in the long term due to their improved diagnostic capability and subsequent reduced costs incurred from the management of clinical complications [15]. What remains unknown is how long Strongyloidesspecific PCR positivity persists in stool samples after antihelminthic treatment and how best to perform post-treatment monitoring to confirm eradication [20,34].

Strongyloides infection can involve any area of the gastrointestinal tract and should be especially considered in the presence of duodenitis. Duodenal aspirates appear to be very sensitive and can be considered in patients with hyperinfection syndrome when it is crucial to make a rapid diagnosis but are more invasive and therefore remain less desirable [35, 62]. Gastrointestinal endoscopy can be utilized for diagnosis but is also an invasive procedure. Endoscopic findings include ulceration (which may be serpiginous), duodenal spasm, bleeding (including subepithelial hemorrhages), erosions, mucosal edema, erythema, thickened duodenal folds, brown discoloration of the mucosa, and megaduodenum [34]. Pustule-like lesions in the colon may represent the process of larvae burying themselves in the mucosa [34]. Microscopic examination of duodenal fluid can be more sensitive than wet mount examination of stool with a reported sensitivity of $76 \%$ [62]. In two-thirds of patients, parasitic forms were found only in duodenal fluid and not in excreted feces [62]. Histopathological examination of duodenal or jejunal biopsy specimens may reveal eosinophilic inflammation, ova, and/or larvae embedded in the lamina propria $[34,62]$.

Imaging studies are not expected to be useful for the diagnosis of uncomplicated Strongyloides infection whereas they may be contributory but not solely diagnostic in disseminated strongyloidiasis and hyperinfection syndrome. Chest $\mathrm{X}$-ray findings in pulmonary strongyloidiasis can be variable due to the different types of bacterial superinfection [62]. Pulmonary infiltrates, when present, may be alveolar or interstitial, diffuse or focal, and unilateral or bilateral [62]. Lung consolidation, cavitation, and abscess formation have been reported [62]. Diffuse bilateral ground glass opacities can be seen on chest $\mathrm{CT}$ in hyperinfection syndrome and DAH. Other imaging studies can be considered based upon the anatomic site of suspected infection to help guide additional diagnostic procedures but these imaging findings would be expected to be nonspecific if performed in isolation.

Because disseminated strongyloidiasis and hyperinfection syndrome are frequently accompanied by secondary bacterial infections, especially from enteric flora, blood cultures should be performed. Additional investigations should be performed depending on clinically suspected sites of infection.

\section{Differential Diagnosis}


The differential diagnosis for peripheral eosinophilia is extensive. Other parasitic infections such as ascariasis, hookworm infections, paragonimiasis, toxocariasis, trichinellosis, and schistosomiasis can also cause eosinophilia and should be considered on the differential diagnosis [70]. It should be noted that coinfection with S. stercoralis and other helminths can occur [70]. Stool microscopy can help establish the diagnosis, acknowledging the aforementioned limited sensitivity of microscopic examinations for the detection of S. stercoralis [15, 70]. Pathogenspecific serologies can also be performed [70]. Apart from parasitic infections, peripheral eosinophilia can be seen in patients with coccidioidomycosis and should be considered in the right clinical context [71]. Other noninfectious causes of eosinophilia include medications and numerous allergic, autoimmune, and neoplastic conditions.

Ascariasis and hookworm infections can cause nonspecific gastrointestinal symptoms with or without eosinophilia [70]. Various gastrointestinal disorders including ulcerative colitis can also be mistaken for gastrointestinal strongyloidiasis [60]. The most helpful distinguishing features of Strongyloides infection from ulcerative colitis are the presence of skip lesions, distal disease attenuation, eosinophil-rich inflammation with or without eosinophilic microabscess formation, relative intact crypt architecture, extracrypt microabscess, and frequent submucosal involvement of the submucosa [60]. A history of corticosteroid therapy, refractory diarrhea, possible rash, pulmonary infiltration, peripheral eosinophilia, and endoscopic findings of right-sided colitis with aphthoid ulcers would strongly favor the diagnosis of strongyloidiasis, especially when they are present together [60].

Pulmonary strongyloidiasis and tropical pulmonary eosinophilia (TPE) due to filarial nematodes such as Wuchereria bancrofti, Brugia malayi, and B. timori can have similar clinical presentations, manifesting with dry cough, wheeze, fatigue, and/or eosinophilia [72]. The peripheral eosinophilia seen in patients with TPE is characteristically pronounced, often exceeding 3,000 cells/ $\mu \mathrm{l}$ [72]. The diagnosis can be confirmed with filarial antibody titers which are usually markedly elevated [72]. Ascariasis and hookworm infections can cause nonspecific pulmonary symptoms with or without eosinophilia [70]. In immunocompromised hosts such as SOT recipients, other causes of ARDS, $\mathrm{DAH}$, and/or diffuse bilateral GGO on chest CT include viral infections (including cytomegalovirus and adenovirus) and the mechanistic target of rapamycin (mTOR) inhibitors sirolimus and everolimus [73-76].

Larva currens must be distinguished from cutaneous larva migrans (CLM), a cutaneous hookworm infection most commonly caused by Ancylostoma braziliense in the Americas [54, 55]. Both are associated with migratory serpiginous lesions that are erythematous, raised, and pruritic $[54,55]$. In contrast, CLM progresses more slowly (approximately 1 to $2 \mathrm{~cm}$ per day), has an angry red appearance, and typically persists for weeks $[54,55]$. Ascariasis and hookworm infections can cause chronic urticaria and/or pruritis $[54,55,70]$.

\section{Treatment}

All persons diagnosed with $S$. stercoralis infection must be treated with curative intent and not just symptomatic improvement [35]. It cannot be overemphasized enough that this is of paramount consequence in the field of SOT since undiagnosed and/or untreated strongyloidiasis of either the donor or recipient can lead to the recipient's demise. Empiric ivermectin can be justified for a patient with epidemiologic risk and a compatible clinical history who is preparing to 
imminently undergo immunosuppression (particularly corticosteroid therapy) in the absence of a confirmed diagnosis of Strongyloides infection, recognizing the costs, limitations, and delays (sometimes more than 2 weeks) of currently available diagnostic assays [35, 48]. Similarly, empiric ivermectin should be given to patients who are strongly suspected to have hyperinfection syndrome based on their epidemiologic risk and clinical presentation because of these diagnostic limitations and the extremely high mortality associated with hyperinfection syndrome. A number of different treatment regimens have been used but a standardized approach has not been established. Treatment options for both uncomplicated and complicated strongyloidiasis in solid organ transplant donors and recipients are summarized in Table 1.

Table 1 Treatment of Strongyloidiasis in Solid Organ Transplant Donors and Recipients

\begin{tabular}{|c|c|c|}
\hline Clinical Syndrome & Preferred Regimen & Alternative Regimens \\
\hline \multirow[t]{2}{*}{$\begin{array}{l}\text { Uncomplicated } \\
\text { (Acute or Chronic) }\end{array}$} & \multirow[t]{2}{*}{$\begin{array}{l}\text { Ivermectin } 200 \mu \mathrm{g} / \mathrm{kg} \text { single } \\
\text { dose PO on } 2 \text { consecutive } \\
\text { days, repeat } 2 \text { weeks later }{ }^{1}\end{array}$} & $\begin{array}{l}\text { Albendazole } 400 \mathrm{mg} \text { PO q12h for } 10-14 \\
\text { days }^{2}\end{array}$ \\
\hline & & Moxidectin $8 \mathrm{mg}$ single dose $\mathrm{PO}^{3}$ \\
\hline \multirow{4}{*}{$\begin{array}{l}\text { Complicated } \\
\text { (Disseminated and/or } \\
\text { Hyperinfection) }\end{array}$} & \multirow[t]{4}{*}{$\begin{array}{l}\text { Ivermectin } 200 \mu \mathrm{g} / \mathrm{kg} \text { single } \\
\text { dose PO q } 24 \mathrm{~h}^{1}\end{array}$} & $\begin{array}{l}\text { Ivermectin } 200 \mu \mathrm{g} / \mathrm{kg} \text { single dose PO } \\
\mathrm{q} 24 \mathrm{~h}^{1}+\text { Albendazole } 400 \mathrm{mg} \text { PO } \mathrm{q} 12 \mathrm{~h}^{2}\end{array}$ \\
\hline & & Ivermectin $200 \mu \mathrm{g} / \mathrm{kg}$ PR q24h \\
\hline & & $\begin{array}{l}\text { Ivermectin } 200 \mu \mathrm{g} / \mathrm{kg} \mathrm{SC} \text { q } 24 \mathrm{~h} \text { in } \\
\text { divided doses into each arm }\end{array}$ \\
\hline & & Albendazole $400 \mathrm{mg}$ PO q12h² \\
\hline
\end{tabular}

Abbreviations: PO, per os or by mouth; PR, per rectum; SC, subcutaneous.

1. Ivermectin is contraindicated in patients with high-grade Loa loa microfilaremia due to risk of encephalopathy.

2. Albendazole should be administered on an empty stomach.

3. Moxidectin is not well-studied but can be considered in situations where ivermectin and albendazole cannot be used for treatment of uncomplicated strongyloidiasis.

4. Treatment should be continued daily until clinical disease resolution and daily specimen microscopy examinations and/or cultures are consistently negative for at least 2 weeks.

5. Ivermectin subcutaneous formulation can be used if PO and PR administration are not possible but requires investigational new drug exemption from the US FDA.

For uncomplicated strongyloidiasis, ivermectin is the treatment of choice because of its effectiveness and tolerability [35, 48]. A single oral dose of ivermectin $200 \mu \mathrm{g} / \mathrm{kg}$ has been reported to achieve a parasitological cure rate of $88 \%$; a second dose improves the cure rate to $96 \%$ in the general population [20]. However, ivermectin's true effectiveness is likely not known because this data is based upon low-sensitivity conventional diagnostic methods [20]. Ivermectin has been administered $200 \mu \mathrm{g} / \mathrm{kg} /$ day orally on 2 days but the ideal timing of the second dose has been controversial. Some experts recommend that these 2 days be consecutive while others argue 
that these 2 days be separated 2 weeks apart [18]. Many reports have shown that these ivermectin regimens failed to attain complete success, presumably because of the 2 to 4 weeks duration of the $S$. stercoralis autoinfection cycle and because ivermectin's activity is limited to the intestinal stages of the parasite leaving the extraintestinal stages unaffected [20]. Considering all of the above, we propose that 4 total doses of ivermectin should be administered (a single oral $200 \mu \mathrm{g} / \mathrm{kg}$ dose on 2 consecutive days, which should then be repeated 2 weeks later) to optimize parasitological cure and treatment success [20]. The treatment course should be repeated if the individual is reexposed to and/or reinfected with $S$. stercoralis. While ivermectin is generally welltolerated, persistently elevated levels of ivermectin in serum and abnormal drug brain level (particularly if given daily in the setting of treatment of hyperinfection syndrome) can potentially lead to severe neurotoxicity even in the absence of genetic or iatrogenic factors known to decrease ivermectin elimination [77]. Drug-drug interactions and drug-food interactions can occur with ivermectin and should be taken into consideration [78]. Ivermectin is contraindicated in patients with high-grade Loa loa microfilaremia because of the risk for encephalopathy; albendazole is preferred in this clinical scenario [48].

Albendazole is an alternative agent for uncomplicated strongyloidiasis that is less effective than ivermectin when administered based upon current treatment recommendations (400 $\mathrm{mg}$ by mouth on an empty stomach twice daily for 3 to 7 days). Therefore, a longer treatment course (10 to 14 days) should be given in order to achieve results comparable to ivermectin $[35,48]$. Thiabendazole had been used in the past but is now rarely used because of its subpar effectiveness ( 67 to $81 \%$ parasitological cure rate) and prohibitive adverse effects (nausea, foulsmelling urine, neuropsychiatric effects, malaise, dizziness, and hypersensitivity reactions including Stevens-Johnson syndrome) $[35,48]$. Moxidectin ( $8 \mathrm{mg}$ by mouth single dose), a wellestablished therapeutic in veterinary medicine that is currently approved by US Food and Drug Administration (FDA) for the treatment of onchocerciasis in people aged 12 years of age and older, is not well-studied but may be a safe and effective option in situations where ivermectin and albendazole cannot be used [19].

Some experts advocate for post-treatment monitoring to assess the effectiveness of treatment of uncomplicated strongyloidiasis. In patients who had positive stool microscopy pre-treatment, follow-up stool examinations 2 to 4 weeks after treatment completion are recommended but their utility is questioned given their low sensitivity and potential for yielding falsely-reassuring negative results [15]. Resolution of peripheral eosinophilia and decrease in antibody titers have also been suggested monitoring parameters but these cannot be considered reliable either since peripheral eosinophilia is variably present and serology can remain positive for an extended duration after treatment [34, 48].

Data is even more limited regarding the preferred management of disseminated strongyloidiasis and hyperinfection syndrome. A strategy of antihelminthic therapy in conjunction with immunosuppression reduction has been traditionally recommended [48]. In SOT recipients, the potential benefits of immunosuppression reduction need to be carefully weighed against the risks of allograft rejection (and its subsequent immune dysfunction and requirement for intensified immunosuppression). Corticosteroids, given their particular implication in promoting accelerated autoinfection and hyperinfection syndrome, should be discontinued if reasonably safe to do so with close monitoring of allograft function but this approach is of questionable benefit [35]. 
In the setting of hyperinfection syndrome, ivermectin $(200 \mu \mathrm{g} / \mathrm{kg} /$ day $)$ is the treatment of choice and should be administered daily by mouth until clinical disease resolution and daily specimen microscopy examinations and/or cultures are consistently negative for at least 2 weeks (correlating to one autoinfection cycle) $[35,48]$. In patients who are unable to tolerate oral ivermectin, alternative routes of administration may be required. In patients with paralytic ileus, ivermectin absorption may be impaired even if administered via nasogastric tube [48, 79]. Rectal ivermectin $(200 \mu \mathrm{g} / \mathrm{kg} / \mathrm{day})$ has been used with occasional success [48, 79]. A veterinary subcutaneous ivermectin formulation $(200 \mu \mathrm{g} / \mathrm{kg} / \mathrm{day}$ in divided doses into each arm), recognized by the Centers for Disease Control and Prevention as a therapeutic option when oral and/or rectal ivermectin administration is not possible, requires an investigational new drug exemption from the US FDA and has been used with mixed results $[48,79,80]$.

Alternative treatment regimens for disseminated strongyloidiasis and hyperinfection syndrome have been published [35, 48, 61]. A combination of ivermectin and albendazole is often considered a reasonable approach given the direness of the situation despite the lack of highquality data from randomized controlled trials or prospective studies comparing this approach to ivermectin monotherapy $[48,61]$. Albendazole is a viable alternative to ivermectin, having been used successfully in hyperinfection syndrome, but may not be as effective [35]. Thiabendazole and mebendazole have also been used with success on a number of occasions but should not be used preferentially over ivermectin or albendazole $[35,61]$. The potential benefit of these less familiar regimens should not be overestimated since there may be a significant publication bias as negative outcomes are less likely to published than positive outcomes. Moxidectin has not yet been evaluated for treatment of hyperinfection syndrome.

In contrast to post-treatment follow-up monitoring of uncomplicated strongyloidiasis, daily surveillance is recommended in the setting of hyperinfection syndrome $[35,48]$. Specimens for monitoring should be obtained from clinically suspected sites of S. stercoralis infection (e.g., stool, respiratory tract, urine, etc.) [35]. Molecular diagnostics such as PCR can be performed if available and it is anticipated that these will likely play a larger role in the future as they become more widely adopted $[14,15,20,34,68]$.

Disseminated strongyloidiasis and hyperinfection syndrome are frequently accompanied by secondary bacterial infections, especially from enteric flora. Appropriate antimicrobial therapy should be tailored to the identified and/or suspected pathogens and site(s) of involvement.

\section{Prognosis}

The overall prognosis for uncomplicated strongyloidiasis is good and patients can live for up to 75 years with chronic strongyloidiasis [31]. Because of their requisite immunosuppression, SOT recipients are more likely to develop disseminated strongyloidiasis and hyperinfection syndrome. These syndromes carry a grim prognosis with mortality approaching $100 \%$ without treatment and still exceeding $25 \%$ despite treatment $[28,41,48]$.

\section{Prevention}

Prevention of strongyloidiasis is mainly by avoiding skin contact with contaminated materials. The vast majority of cases could be averted by wearing shoes and avoiding barefoot walking on contaminated soil. Appropriate hand hygiene and environmental disinfection could help prevent 
institutional outbreaks [36, 37]. Avoidance of close contact with potentially infected body fluids of patients with hyperinfection syndrome can prevent person-to-person transmission of Strongyloides filariform larvae [38]. Screening and treatment of SOT donors before organ procurement may prevent donor-derived infections in the SOT recipient [6-10]. In situations where pre-transplant screening of the donor and/or the recipient is not performed, ivermectin can be empirically administered to the SOT recipient in certain circumstances to prevent posttransplant disseminated strongyloidiasis and hyperinfection syndrome.

\section{Acknowledgments}

None.

\section{Author Contributions}

Multani A - Conceptualization, Writing (including Original Draft Preparation, Reviewing, and Editing); Deresinski S - Conceptualization, Writing (including Reviewing and Editing), Supervision.

\section{Funding}

None.

\section{Competing Interests}

The authors have declared that no competing interests exist.

\section{References}

1. Genta RM. Global prevalence of strongyloidiasis: critical review with epidemiologic insights into the prevention of disseminated disease. Rev Infect Dis. 1989; 11: 755-767.

2. Puthiyakunnon S, Boddu S, Li Y, Zhou X, Wang C, Li J, et al. Strongyloidiasis--an insight into its global prevalence and management. PLoS Negl Trop Dis. 2014; 8: e3018.

3. Hoy WE, Roberts NJ Jr, Bryson MF, Bowles C, Lee JC, Rivero AJ, et al. Transmission of strongyloidiasis by kidney transplant? Disseminated strongyloidiasis in both recipients of kidney allografts from a single cadaver donor. JAMA. 1981; 246: 1937-1939.

4. Marty FM. Strongyloides hyperinfection syndrome and transplantation: a preventable, frequently fatal infection. Transpl Infect Dis. 2009; 11: 97-99.

5. Rodriguez-Hernandez MJ, Ruiz-Perez-Pipaon M, Cañas E, Bernal C, Gavilan F. Strongyloides stercoralis hyperinfection transmitted by liver allograft in a transplant recipient. Am J Transplant. 2009; 9: 2637-2640.

6. Hamilton KW, Abt PL, Rosenbach MA, Bleicher MB, Levine MS, Mehta J, et al. Donor-derived Strongyloides stercoralis infections in renal transplant recipients. Transplantation. 2011; 91: 1019-1024.

7. Weiser JA, Scully BE, Bulman WA, Husain S, Grossman ME. Periumbilical parasitic thumbprint purpura: strongyloides hyperinfection syndrome acquired from a cadaveric renal transplant. Transpl Infect Dis. 2011; 13: 58-62. 
8. Centers for Disease Control and Prevention (CDC). Transmission of Strongyloides stercoralis through transplantation of solid organs--Pennsylvania, 2012. MMWR Morb Mortal Wkly Rep. 2013; 62: 264-266.

9. Abanyie FA, Gray EB, Delli Carpini KW, Yanofsky A, McAuliffe I, Rana M, et al. Donor-derived Strongyloides stercoralis infection in solid organ transplant recipients in the United States, 2009-2013. Am J Transplant. 2015; 15: 1369-1375.

10. Kim JH, Kim DS, Yoon YK, Sohn JW, Kim MJ. Donor-Derived Strongyloidiasis Infection in Solid Organ Transplant Recipients: A Review and Pooled Analysis. Transplant Proc. 2016; 48: 24422449.

11. Nordheim E, Olafsson Storr $\varnothing$ M, Natvik AK, Birkeland Kro G, Midtvedt K, Varberg Reisaeter A, et al. Donor-derived strongyloidiasis after organ transplantation in Norway. Transpl Infect Dis. 2018; e13008.

12. Loutfy MR, Wilson M, Keystone JS, Kain KC. Serology and eosinophil count in the diagnosis and management of strongyloidiasis in a non-endemic area. Am J Trop Med Hyg. 2002; 66: 749-752.

13. Boulware DR, Stauffer WM, Hendel-Paterson BR, Rocha JLL, Seet RC-S, Summer AP, et al. Maltreatment of Strongyloides infection: case series and worldwide physicians-in-training survey. Am J Med. 2007; 120: 545.e1-8.

14. Sharifdini M, Mirhendi $H$, Ashrafi K, Hosseini M, Mohebali M, Khodadadi $H$, et al. Comparison of Nested Polymerase Chain Reaction and Real-Time Polymerase Chain Reaction with Parasitological Methods for Detection of Strongyloides stercoralis in Human Fecal Samples. Am J Trop Med Hyg. 2015; 93: 1285-1291.

15. Repetto SA, Ruybal P, Solana ME, López C, Berini CA, Alba Soto CD, et al. Comparison between PCR and larvae visualization methods for diagnosis of Strongyloides stercoralis out of endemic area: A proposed algorithm. Acta Trop. 2016; 157: 169-177.

16. Gómez-Junyent J, Paredes-Zapata D, de las Parras ER, González-Costello J, Ruiz-Arranz Á, Cañizares $R$, et al. Real-Time Polymerase Chain Reaction in Stool Detects Transmission of Strongyloides stercoralis from an Infected Donor to Solid Organ Transplant Recipients. Am J Trop Med Hyg. 2016; 94: 897-899.

17. Ashraf M, Gue CL, Baddour LM. Strongyloidiasis Refractory to Treatment With Ivermectin. Am J Med Sci. 1996; 311: 178-179.

18. Zaha O, Hirata T, Kinjo F, Saito A, Fukuhara H. Efficacy of ivermectin for chronic strongyloidiasis: two single doses given 2 weeks apart. J Infect Chemother. 2002; 8: 94-98.

19. Barda B, Sayasone S, Phongluxa K, Xayavong S, Keoduangsy K, Odermatt P, et al. Efficacy of moxidectin versus ivermectin against Strongyloides stercoralis infections: a randomized controlled non-inferiority trial. Clin Infect Dis. 2017; 65: 276-281.

20. Repetto SA, Ruybal P, Batalla E, López C, Fridman V, Sierra M, et al. Strongyloidiasis Outside Endemic Areas: Long-term Parasitological and Clinical Follow-up After Ivermectin Treatment. Clin Infect Dis. 2018; 66: 1558-1565.

21. Schär F, Trostdorf U, Giardina F, Khieu V, Muth S, Marti H, et al. Strongyloides stercoralis: Global Distribution and Risk Factors. PLoS Negl Trop Dis. 2013; 7: e2288.

22. Levi ME, Kumar D, Green M, Ison MG, Kaul D, Michaels MG, et al. Considerations for screening live kidney donors for endemic infections: a viewpoint on the UNOS policy. Am J Transplant. 2014; 14: 1003-1011. 
23. Croker C, Reporter R, Redelings M, Mascola L. Strongyloidiasis-related deaths in the United States, 1991-2006. Am J Trop Med Hyg. 2010; 83: 422-426.

24. Centers for Disease Control and Prevention. Notes from the field: Strongyloidiasis in a rural setting--Southeastern Kentucky, 2013. MMWR Morb Mortal Wkly Rep. 2013; 62: 843.

25. Ashford RW, Barnish G, Viney ME. Strongyloides fuelleborni kellyi: infection and disease in Papua New Guinea. Parasitol Today. 1992; 8: 314-318.

26. Thanchomnang T, Intapan PM, Sanpool O, Rodpai R, Tourtip S, Yahom S, et al. First molecular identification and genetic diversity of Strongyloides stercoralis and Strongyloides fuelleborni in human communities having contact with long-tailed macaques in Thailand. Parasitol Res. 2017; 116: 1917-1923.

27. Rutherford AM. Reflections on the passage of Strongyloides fülleborni to human infants in mothers' milk. Trop Doct. 1981; 11: 184-185.

28. Grove DI. Human strongyloidiasis. Adv Parasitol. 1996; 38: 251-309.

29. Gonçalves ALR, Machado GA, Gonçalves-Pires MRF, Ferreira-Júnior A, Silva DAO, Costa-Cruz $J M$. Evaluation of strongyloidiasis in kennel dogs and keepers by parasitological and serological assays. Vet Parasitol. 2007; 147: 132-139.

30. Pelletier LL Jr, Baker CB, Gam AA, Nutman TB, Neva FA. Diagnosis and evaluation of treatment of chronic strongyloidiasis in ex-prisoners of war. J Infect Dis. 1988; 157: 573-576.

31. Prendki V, Fenaux $P$, Durand $R$, Thellier $M$, Bouchaud $O$. Strongyloidiasis in man 75 years after initial exposure. Emerg Infect Dis. 2011; 17: 931-932.

32. Chu E, Whitlock WL, Dietrich RA. Pulmonary hyperinfection syndrome with Strongyloides stercoralis. Chest. 1990; 97: 1475-1477.

33. Siegel MO, Simon GL. Is human immunodeficiency virus infection a risk factor for Strongyloides stercoralis hyperinfection and dissemination. PLoS Negl Trop Dis. 2012; 6: e1581.

34. Requena-Méndez A, Chiodini P, Bisoffi Z, Buonfrate D, Gotuzzo E, Muñoz J. The laboratory diagnosis and follow up of strongyloidiasis: a systematic review. PLoS Negl Trop Dis. 2013; 7: e2002.

35. Keiser PB, Nutman TB. Strongyloides stercoralis in the Immunocompromised Population. Clin Microbiol Rev. 2004; 17: 208-217.

36. Centers for Disease Control and Prevention. Notes from the field: strongyloides infection among patients at a long-term care facility--Florida, 2010-2012. MMWR Morb Mortal Wkly Rep. 2013; 62: 844.

37. Jones JM, Hill C, Briggs G, Gray E, Handali S, McAuliffe I, et al. Notes from the Field: Strongyloidiasis at a Long-Term-Care Facility for the Developmentally Disabled - Arizona, 2015. MMWR Morb Mortal Wkly Rep. 2016; 65: 608-609.

38. Czachor JS, Jonas AP. Transmission of Strongyloides steracolis person to person. J Travel Med. 2000; 7: 211-212.

39. Sorvillo F, Mori K, Sewake W, Fishman L. Sexual transmission of Strongyloides stercoralis among homosexual men. Br J Vener Dis. 1983; 59: 342.

40. Brown RC, Girardeau HF. Transmammary passage of Strongyloides sp. larvae in the human host. Am J Trop Med Hyg. 1977; 26: 215-219.

41. Fardet L, Généreau T, Poirot J-L, Guidet B, Kettaneh A, Cabane J. Severe strongyloidiasis in corticosteroid-treated patients: case series and literature review. J Infect. 2007; 54: 18-27. 
42. Ghosh K, Ghosh K. Strongyloides stercoralis septicaemia following steroid therapy for eosinophilia: report of three cases. Trans R Soc Trop Med Hyg. 2007; 101: 1163-1165.

43. Marcos LA, Terashima A, Dupont HL, Gotuzzo E. Strongyloides hyperinfection syndrome: an emerging global infectious disease. Trans R Soc Trop Med Hyg. 2008; 102: 314-318.

44. Neva FA. Biology and immunology of human strongyloidiasis. J Infect Dis. 1986; 153: 397-406.

45. Genta RM. Dysregulation of strongyloidiasis: a new hypothesis. Clin Microbiol Rev. 1992; 5: 345-355.

46. Mansfield LS, Niamatali S, Bhopale V, Volk S, Smith G, Lok JB, et al. Strongyloides stercoralis: maintenance of exceedingly chronic infections. Am J Trop Med Hyg. 1996; 55: 617-624.

47. Siddiqui AA, Stanley CS, Skelly PJ, Berk SL. A cDNA encoding a nuclear hormone receptor of the steroid/thyroid hormone-receptor superfamily from the human parasitic nematode Strongyloides stercoralis. Parasitol Res. 2000; 86: 24-29.

48. Mejia R, Nutman TB. Screening, prevention, and treatment for hyperinfection syndrome and disseminated infections caused by Strongyloides stercoralis. Curr Opin Infect Dis. 2012; 25: 458-463.

49. Schad GA. Cyclosporine may eliminate the threat of overwhelming strongyloidiasis in immunosuppressed patients. J Infect Dis. 1986; 153: 178.

50. Rosen A, Ison MG. Screening of living organ donors for endemic infections: Understanding the challenges and benefits of enhanced screening. Transpl Infect Dis. 2016. doi.org/10.1111/tid.12633

51. Schwartz BS, Mawhorter SD, AST Infectious Diseases Community of Practice. Parasitic infections in solid organ transplantation. Am J Transplant. 2013; 13: 280-303.

52. Abanyie FA, Valice E, Delli Carpini KW, Gray EB, McAuliffe I, Chin-Hong PV, et al. Organ donor screening practices for Strongyloides stercoralis infection among US organ procurement organizations. Transpl Infect Dis. 2018; 20: e12865.

53. Freedman DO. Experimental infection of human subject with Strongyloides species. Rev Infect Dis. 1991; 13: 1221-1226.

54. Meinking TL, Burkhart CN, Burkhart CG. Changing paradigms in parasitic infections: common dermatological helminthic infections and cutaneous myiasis. Clin Dermatol. 2003; 21: 407416.

55. Arthur RP, Shelley WB. Larva currens; a distinctive variant of cutaneous larva migrans due to Strongyloides stercoralis. AMA Arch Derm. 1958; 78: 186-190.

56. Sen P, Gil C, Estrellas B, Middleton JR. Corticosteroid-induced asthma: a manifestation of limited hyperinfection syndrome due to Strongyloides stercoralis. South Med J. 1995; 88: 923-927.

57. Salles F, Bacellar A, Amorim M, Orge G, Sundberg M, Lima M, et al. Treatment of strongyloidiasis in HTLV-1 and Strongyloides stercoralis coinfected patients is associated with increased TNF $\alpha$ and decreased soluble IL2 receptor levels. Trans R Soc Trop Med Hyg. 2013; 107: 526-529.

58. Wong TY, Szeto CC, Lai FF, Mak CK, Li PK. Nephrotic syndrome in strongyloidiasis: remission after eradication with anthelmintic agents. Nephron. 1998; 79: 333-336.

59. Lai C-P, Hsu Y-H, Wang J-H, Lin C-M. Strongyloides stercoralis infection with bloody pericardial effusion in a non-immunosuppressed patient. Circ J. 2002; 66: 613-614. 
60. Qu Z, Kundu UR, Abadeer RA, Wanger A. Strongyloides colitis is a lethal mimic of ulcerative colitis: the key morphologic differential diagnosis. Hum Pathol. 2009; 40: 572-577.

61. Segarra-Newnham M. Manifestations, diagnosis, and treatment of Strongyloides stercoralis infection. Ann Pharmacother. 2007; 41: 1992-2001.

62. Siddiqui AA, Berk SL. Diagnosis of Strongyloides stercoralis infection. Clin Infect Dis. 2001; 33: 1040-1047.

63. Robinson RD, Lindo JF, Neva FA, Gam AA, Vogel P, Terry SI, et al. Immunoepidemiologic studies of Strongyloides stercoralis and human T lymphotropic virus type I infections in Jamaica. J Infect Dis. 1994; 169: 692-696.

64. Higashiarakawa M, Hirata $T$, Tanaka $T$, Parrott $G$, Kinjo $T$, Naka H, et al. Normal serum IgE levels and eosinophil counts exhibited during Strongyloides stercoralis infection. Parasitol Int. 2017; 66: 807-812.

65. Nielsen PB, Mojon M. Improved diagnosis of strongyloides stercoralis by seven consecutive stool specimens. Zentralbl Bakteriol Mikrobiol Hyg A. 1987; 263: 616-618.

66. Abdalla J, Saad M, Myers JW, Moorman JP. An elderly man with immunosuppression, shortness of breath, and eosinophilia. Clin Infect Dis. 2005; 40: 1464, 1535-1536.

67. Repetto SA, Alba Soto CD, Cazorla SI, Tayeldin ML, Cuello S, Lasala MB, et al. An improved DNA isolation technique for PCR detection of Strongyloides stercoralis in stool samples. Acta Trop. 2013; 126: 110-114.

68. Barda B, Wampfler R, Sayasone S, Phongluxa K, Xayavong S, Keoduangsy K, et al. Evaluation of Two DNA Extraction Methods for Detection of Strongyloides stercoralis Infection. J Clin Microbiol. 2018; 56. doi.org/10.1128/JCM.01941-17

69. Konnick EQ, Chow S-K, Reder NP, Sengupta DJ, Hoogestraat DR, Pottinger PS, et al. Incidental identification of Strongyloides stercoralis infection by broad-range 28S rDNA gene sequencing in a patient with a hematolymphoid malignancy. Diagn Microbiol Infect Dis. 2016; 86: 362364.

70. Jourdan PM, Lamberton PHL, Fenwick A, Addiss DG. Soil-transmitted helminth infections. Lancet. 2017. doi.org/10.1016/S0140-6736(17)31930-X

71. Galgiani JN, Ampel NM, Blair JE, Catanzaro A, Geertsma F, Hoover SE, et al. 2016 Infectious Diseases Society of America (IDSA) Clinical Practice Guideline for the Treatment of Coccidioidomycosis. Clin Infect Dis. 2016; 63: e112-e146.

72. Ottesen EA, Nutman TB. Tropical pulmonary eosinophilia. Annu Rev Med. 1992;43:417-24.

73. Pham P-TT, Pham P-CT, Danovitch GM, Ross DJ, Gritsch HA, Kendrick EA, et al. Sirolimusassociated pulmonary toxicity. Transplantation. 2004; 77: 1215-1220.

74. Champion L, Stern $M$, Israël-Biet $D$, Mamzer-Bruneel $M-F$, Peraldi $M-N$, Kreis $H$, et al. Brief communication: sirolimus-associated pneumonitis: 24 cases in renal transplant recipients. Ann Intern Med. 2006; 144: 505-509.

75. Vandewiele B, Vandecasteele SJ, Vanwalleghem L, De Vriese AS. Diffuse alveolar hemorrhage induced by everolimus. Chest. 2010; 137: 456-459.

76. von Ranke FM, Zanetti G, Hochhegger B, Marchiori E. Infectious diseases causing diffuse alveolar hemorrhage in immunocompetent patients: a state-of-the-art review. Lung. 2013; 191: 9-18. 
77. van Westerloo DJ, Landman GW, Prichard R, Lespine A, Visser LG. Persistent coma in Strongyloides hyperinfection syndrome associated with persistently increased ivermectin levels. Clin Infect Dis. 2014; 58: 143-144.

78. González Canga A, Sahagún Prieto AM, Diez Liébana MJ, Fernández Martínez N, Sierra Vega M, García Vieitez JJ. The pharmacokinetics and interactions of ivermectin in humans--a minireview. AAPS J. 2008; 10: 42-46.

79. Zeitler K, Jariwala R, Restrepo-Jaramillo R, Kapadia S, Casanas B, Alrabaa S, et al. Successful use of subcutaneous ivermectin for the treatment of Strongyloides stercoralis hyperinfection in the setting of small bowel obstruction and paralytic ileus in the immunocompromised population. BMJ Case Rep. 2018. doi.org/10.1136/bcr-2017-223138

80. Lichtenberger P, Rosa-Cunha I, Morris M, Nishida S, Akpinar E, Gaitan J, et al. Hyperinfection strongyloidiasis in a liver transplant recipient treated with parenteral ivermectin. Transpl Infect Dis. 2009; 11: 137-142.

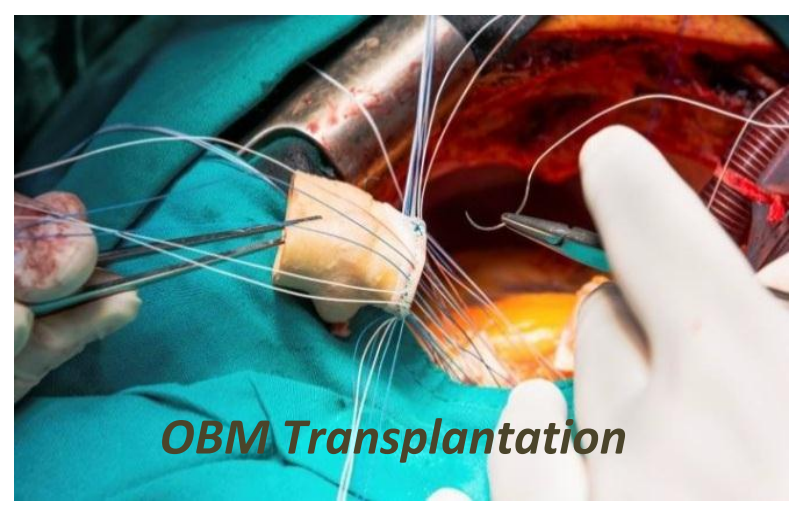

Enjoy OBM Transplantation by:

1. Submitting a manuscript

2. Joining in volunteer reviewer bank

3. Joining Editorial Board

4. Guest editing a special issue

For more details, please visit:

http://www.lidsen.com/journals/transplantation 